\title{
Rapid Amygdala Kindling Causes Motor Seizure and Comorbidity of Anxiety- and Depression-Like Behaviors in Rats
}

\author{
Shang-Der Chen ${ }^{1,2}$, Yu-Lin Wang ${ }^{3}$, Sheng-Fu Liang ${ }^{3,4}$ and Fu-Zen Shaw ${ }^{5 *}$ \\ ${ }^{1}$ Department of Neurology, Kaohsiung Chang Gung Memorial Hospital, Chang Gung University College of Medicine, \\ Kaohsiung, Taiwan, ${ }^{2}$ Center for Translational Research in Biomedical Science, Kaohsiung Chang Gung Memorial Hospital, \\ Chang Gung University College of Medicine, Kaohsiung, Taiwan, ${ }^{3}$ Department of Computer Science and Information \\ Engineering, National Cheng Kung University, Tainan, Taiwan, ${ }^{4}$ Institute of Medical Informatics, National Cheng Kung \\ University, Tainan, Taiwan, ${ }^{5}$ Department of Psychology, National Cheng Kung University, Tainan, Taiwan
}

OPEN ACCESS

Edited by: James P. Herman, University of Cincinnati, USA

Reviewed by: Dimitri De Bundel, Vrije Universiteit Brussel, Belgium Michael Arthur Van Der Kooij, Johannes Gutenberg University Mainz, Germany

${ }^{*}$ Correspondence:

Fu-Zen Shaw fzshaw@gmail.com

Received: 09 January 2016 Accepted: 08 June 2016 Published: 22 June 2016

Citation: Chen S-D, Wang Y-L, Liang S-F and Shaw F-Z (2016) Rapid Amygdala Kindling Causes Motor Seizure and Comorbidity of Anxiety- and Depression-Like Behaviors in Rats. Front. Behav. Neurosci. 10:129. doi: 10.3389/fnbeh.2016.00129
Amygdala kindling is a model of temporal lobe epilepsy (TLE) with convulsion. The rapid amygdala kindling has an advantage on quick development of motor seizures and for antiepileptic drugs screening. The rapid amygdala kindling causes epileptogenesis accompanied by an anxiolytic response in early isolation of rat pups or depressive behavior in immature rats. However, the effect of rapid amygdala kindling on comorbidity of anxiety- and depression-like behaviors is unexplored in adult rats with normal breeding. In the present study, 40 amygdala stimulations given within 2 days were applied in adult Wistar rats. Afterdischarge (AD) and seizure stage were recorded throughout the amygdala kindling. Anxiety-like behaviors were evaluated by the elevated plus maze (EPM) test and open field (OF) test, whereas depression-like behaviors were assessed by the forced swim (FS) and sucrose consumption (SC) tests. A tonic-clonic convulsion was provoked in the kindle group. Rapid amygdala kindling resulted in a significantly lower frequency entering an open area of either open arms of the EPM or the central zone of an OF, lower sucrose intake, and longer immobility of the FS test in the kindle group. Our results suggest that rapid amygdala kindling elicited severe motor seizures comorbid with anxiety- and depression-like behaviors.

Keywords: amygdala kindling, afterdischarge, anxiety, depression, anhedonia

\section{INTRODUCTION}

Temporal lobe epilepsy (TLE) is the most drug-resistant type of adult focal epilepsy. A considerable portion of TLE patients $(20-50 \%)$ have comorbidity of anxiety and/or depression (Kanner, 2007; Desai et al., 2010). The epileptic focus in TLE patients or TLE-like animal models often resides in a mesial temporal structure, such as amygdala, hippocampus, or both. Previous studies have indicated that the amygdala is a critical component of the abnormal circuitry underlying temporal lobe seizures (Goddard, 1983; Pitkänen et al., 1998). The amygdala is among the more susceptible regions to kindling (Kalynchuk, 2000; Morimoto et al., 2004). Thus, amygdala kindling is a popular experimental 
model for TLE with partial-onset and secondarily generalized convulsion (Goddard, 1983).

Conventional amygdala kindling (Goddard, 1983; Lothman and Williamson, 1994), where two electrical stimulations are delivered per day, has been investigated on the process of epileptogenesis and changes in interictal emotionality (Kalynchuk, 2000). The amygdala is a part of the limbic system and is associated with the modulation of emotion and fear. Kindling of the right-side amygdala of animals elicits anxietylike behavior (Adamec and Morgan, 1994; Helfer et al., 1996; Hannesson et al., 2008). Conventional amygdala kindling did not aggravate depression-like behaviors, such as anhedonia or despair mood (Helfer et al., 1996; Wintink et al., 2003; Adamec et al., 2004). Lacking comorbidity of depression-like behavior in the conventional kindling model strikingly differs from observations in TLE patients (Kanner, 2007) or other TLElike animal models, e.g., kainate-induced model (Tchekalarova et al., 2013) or pilocarpine-induced model (Mazarati et al., 2008).

Rapid amygdala kindling, where up to 24 stimulations are given each day (Lothman et al., 1985; Lothman and Williamson, 1994), is a variation of the conventional kindling model with the advantage that kindling can occur over few days and shows neuronal plasticity in the limbic region (Ebert and Löscher, 1995; Smith et al., 2005). The rapid kindling process has been used to assess anticonvulsants or alternative therapeutics (De Smedt et al., 2007; Shahpari et al., 2012; Shojaei et al., 2014). Spike patterns of epileptiform discharges with regard to the severity of motor seizures have a similar development between conventional and rapid amygdala kindling (Wang et al., 2016). However, rapid kindling of the amygdala causes epileptogenesis accompanied by anxiolytic response in early isolation of rat pups (Jones et al., 2009) or depressive behavior in immature rats (Mazarati et al., 2007). Phenomena of psychiatric comorbidity in the rapid kindling model seem to differ from those of conventional kindling model in adult rats (Adamec and Morgan, 1994; Helfer et al., 1996; Wintink et al., 2003; Adamec et al., 2004; Hannesson et al., 2008) or patients with TLE (Kanner, 2007; Desai et al., 2010). These controversial results may arise from using a specific group (immature or early separated stress) in the rapid kindling model. To our knowledge, emotional dysfunctions of the rapid kindling model in adult rats are still unknown.

In the present study, the rapid amygdala kindling was performed in adult rats. We hypothesized that rapid amygdala kindling caused epileptiform activity accompanied by anxietyand depression-like behaviors as similar to most TLE patients with psychiatric comorbidity. The results may advance our understanding in progression of epileptiform activity and emotional disturbance through rapid amygdala kindling.

\section{MATERIALS AND METHODS}

Adult male Wistar rats (8-15 weeks, $350-500$ g) were used. Animals were maintained in a sound-attenuated room under a 12-h light/dark cycle (lights on at 06:00-18:00) with food and water available ad libitum. Rats were randomly assigned to the kindle and control groups. The experimental procedures were reviewed and approved by the Institutional Animal Care and Use Committee. All experiments complied with guidelines recommended by NIH (USA) on the ethical use of animals.

\section{Surgery}

Rats were anesthetized by sodium pentobarbital ( $50 \mathrm{mg} / \mathrm{kg}$, i.p.). Subsequently, the dorsal surface of the head was shaved, and the rat was placed in a standard stereotaxic apparatus. A midline incision was made for placing stainless steel screws and Tefloninsulated 0.2-mm diameter stainless steel microwires (\#791600, A-M Systems, Sequim, WA, USA). Animals were equipped with stainless steel screws connected by insulated wires to a microconnector for electrocorticographic (ECoG) recordings. Screw electrodes were bilaterally implanted over the area of the frontal cortex ( $2 \mathrm{~mm}$ anterior, $2.5-3.5 \mathrm{~mm}$ lateral from the bregma). Three stainless steel microwires, two for stimulation and one for recording, were implanted into the right basolateral amygdala $(2.6 \mathrm{~mm}$ posterior and $4.8 \mathrm{~mm}$ lateral from the bregma, $8.5 \mathrm{~mm}$ ventral from the surface). The stimulated microwires were made of $0.5 \mathrm{~mm}$ tip exposure and the tip separation of $0.5 \mathrm{~mm}$ (Helfer et al., 1996). Two twisted microwires for recording were implanted into the left basolateral amygdala. A ground electrode was implanted $2 \mathrm{~mm}$ caudal to lambda. Screw electrode and microwire assemblies were attached to the skull with dental acrylic. Animals were given antibiotic (chlortetracycline) after suturing and housed individually in cages for a recovery period of $>1$ week.

\section{Kindling}

Stimulation of the right-side amygdala was applied by a constant current stimulator (Grass 48, Grass Technique, West Warwick, RI, USA). Activities of bilateral frontal cortices and amygdala were recorded during the kindling. On the pre-kindling period, a $2-\mathrm{s}, 80-\mathrm{Hz}$ monophasic square-wave stimulus of $1 \mathrm{~ms}$ per pulse was used to determine the afterdischarge (AD) threshold. The stimulus intensity began at $50 \mu \mathrm{A}$, and was subsequently increased by $25 \mu \mathrm{A}$ steps every $30 \mathrm{~min}$ until at least 5 -s $\mathrm{AD}$ was elicited. Therefore, the intensity that $\geq 2$ of three stimulations produced $\mathrm{ADs}$ was defined as the $\mathrm{AD}$ threshold.

One day after determination of the $\mathrm{AD}$ threshold, amygdala kindling was carried out. Stimulations of $200 \mu \mathrm{A}, 80 \mathrm{~Hz}, 1 \mathrm{~ms}$ pulse width for a total duration of $2 \mathrm{~s}$ were delivered from an isolated constant current stimulator. Two consecutive kindling stimulations were separated by $30 \mathrm{~min}$, and a maximum of 20 stimulations were given daily (Racine et al., 1973; Wang et al., 2016). The intensity of the stimulated current for kindling was set at $200 \mu \mathrm{A}$ to control the degree of current spread into adjacent brain regions (Ehlers and Koob, 1985). The behavioral stages observed after each stimulus were classified using Racine's standard 5-stage scale (Racine, 1972): stage 1, facial movements; stage 2, rhythmic head movements, head nodding; stage 3, unilateral forelimb clonus; stage 4, bilateral forelimb clonus and rearing; stage 5, falling and tonic-clonic convulsion. $\mathrm{AD}$ duration was the total time of epileptiform 


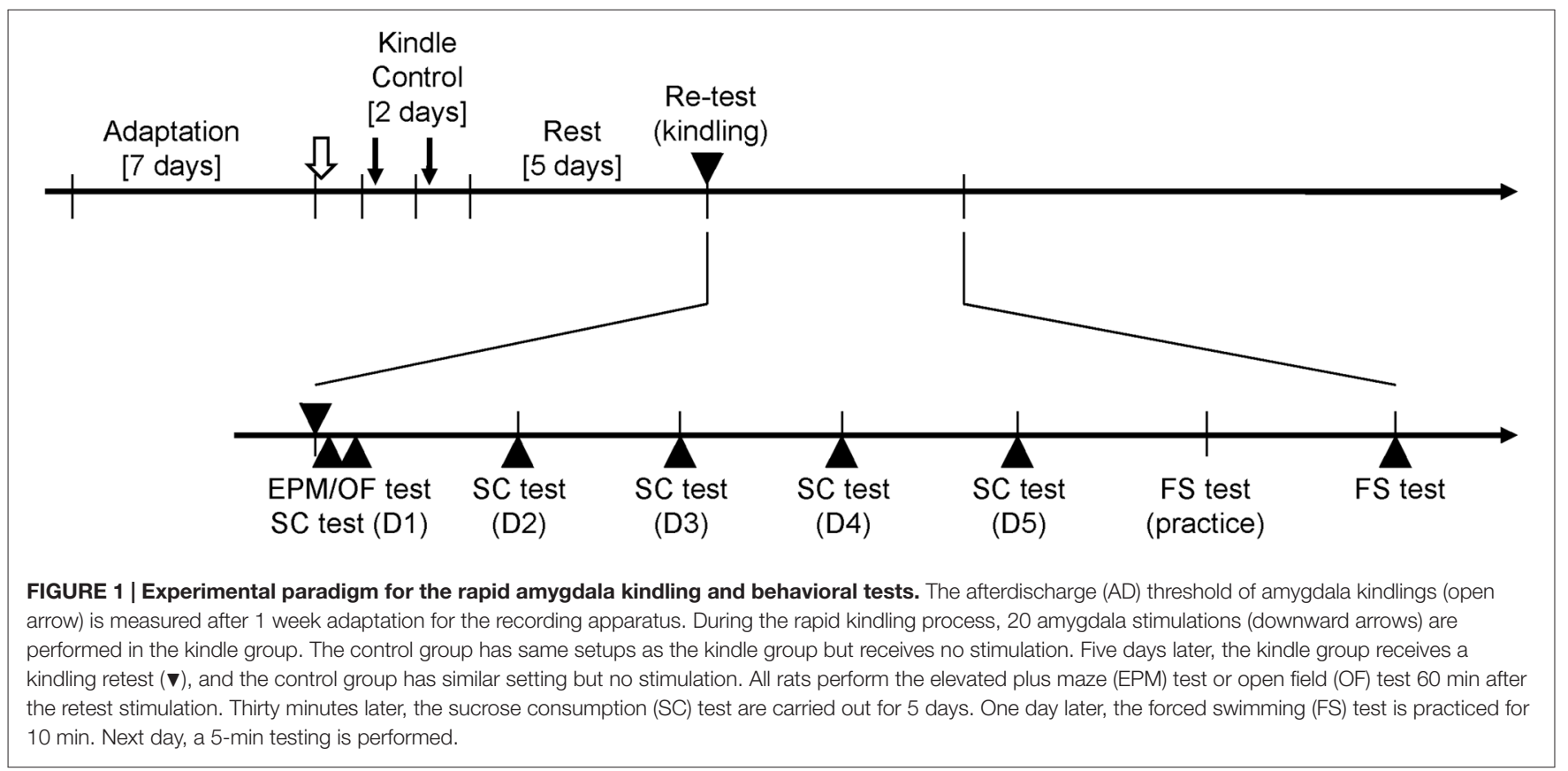

activities at the stimulated amygdala electrode, including the stimulation period.

\section{Recording of Brain Activities}

Bilateral frontal ECoGs and field potentials of bilateral basolateral nuclei of the amygdala were amplified and filtered (0.7-300 Hz) through a multichannel amplifier (Shaw et al., 2002). A grounded metal plate was placed under the recording chamber to reduce electromagnetic interference (Shaw et al., 2003). The amplified signals were digitized at 1000 samples/s (USB6009, National Instruments, TX, USA) and stored on a hard disk for offline analysis. The entire software for data acquisition and analysis was under LabVIEW platform (National Instruments, TX, USA).

\section{Experimental Procedure}

Two weeks after surgery, all rats were placed in a recording room. To allow rats to habituate the experimental setting, each rat was placed in the recording apparatus $30 \mathrm{~min} /$ day for 7 days. The experiments of determining $\mathrm{AD}$ thresholds and all behavioral tests were performed at 13:00-17:00 to minimize circadian influences. AD thresholds of all rats were determined first. Thereafter, 20 amygdala stimuli were repeatedly done in the kindle group for 2 days. In the control group, all rats had the same setup as the kindle group did except for the amygdala stimulation. Five days later, a $200-\mu$ A stimulation was employed to ascertain Racine's stage in the kindle group, whereas the control group was set with a connector assembly for $10 \mathrm{~min}$ without a re-test stimulation. The rat was excluded if Racine's score of the re-testing stimulation was not same as the Racine's score of the 40th stimulation. Sixty minutes later, the elevated plus maze (EPM) test was performed followed by the sucrose consumption (SC) test with interval of $30 \mathrm{~min}$. One day later, the
SC test was performed daily for 4 days. Subsequently, a 15-min habituation was done first, and a 5 -min forced swim (FS) test next day. A schematic flowchart of the entire experimental procedure is shown in Figure 1.

In addition, we carried out the second experiment for the open field (OF) test. The procedure of the second experiment was similar to the first experiment. The kindle group performed the OF test $60 \mathrm{~min}$ after the re-testing kindling stimulation.

\section{Behavioral Test}

Three behavioral tests were used to evaluate the anxiety- and depression-like behaviors of rats (Jones et al., 2008; Huang et al., 2012). The EPM test and OF test are used to measure anxious status. The FS test is used to assess the duration of immobility, which is the experimental analog of a despair-like status. The SC test is a measure of the "hedonic" state of an animal, or the ability to experience pleasure. A decreased sensitivity to reward (or anhedonia) is a fundamental feature of clinical depression.

\section{Sucrose Consumption Test}

In the SC test, each rat was placed in a test cage identical to its home cage. Consumption of a $20 \%$ sucrose solution was recorded for $15 \mathrm{~min}$. Sucrose intake was measured by reweighing a preweighed bottle at the end of the test. Prior to testing, rats were not deprived of food and water. In this study, fluid intake over 5 days was used for statistical evaluation of differences in the two groups of rats. Decreased sucrose intake, i.e., anhedonia, is a validated index of a depression-like state (Jones et al., 2008).

\section{Forced Swim Test}

Apparatus of the FS test was a plastic cylinder $47 \mathrm{~cm}$ in height with a $38-\mathrm{cm}$ inside diameter containing $38 \mathrm{~cm}$ of water at 
$25 \pm 1^{\circ} \mathrm{C}$. The FS test consisted of two phases. Rats were individually forced to swim in a plastic cylinder for $15 \mathrm{~min}$ on the first day. The 5 -min test sessions began $24 \mathrm{~h}$ later. The duration of immobility, including passive swimming, was measured. The criterion for passive swimming was floating vertically in the water while making only those movements necessary to keep the head above the water. The active swimming represented more vigorous activity than swimming: strong movement of all four limbs, jumping, etc. Increased immobility in the forced swimming (FS) test is indicative of depression-like behavior (Cryan et al., 2005).

\section{Elevated Plus Maze Test}

The EPM consisted of black polypropylene plastic which was elevated $68 \mathrm{~cm}$ above the floor. Each maze arm extended $45 \mathrm{~cm}$ from the junction area, which measured $13 \times 13 \mathrm{~cm}$. The open and closed arms (CAs) were $13 \mathrm{~cm}$ wide, and the closed maze arms had walls extending $25 \mathrm{~cm}$ from the junction area. During testing, each rat was placed in the central square facing an open arm (OA) and was allowed 5 min to freely explore the maze. The alleys of the maze were thoroughly cleaned with an ethanol solution (60\% volume) after the removal of each rat. Time spent in OAs and the numbers of open and CA entries were measured. The percentage of entries in OAs was calculated according to the following formula: percentage of OA entry $=$ (number of entries into the OAs/number of open $+\mathrm{CA}$ entries $) \times 100$. Either the percentage of venturing into OAs and/or the amount of time spent in OAs are validated for anxiety (Rodgers and Dalvi, 1997).

\section{Open Field Test}

The test cage was comprised of black acrylic plastic that formed a square $99 \times 99 \mathrm{~cm}$ with a wall height of $45 \mathrm{~cm}$. The box was divided into nine equal squares measuring $33 \times 33 \mathrm{~cm}$. Recording was done in a room illuminated by a ceiling red fluorescent light $(40 \mathrm{~W})$. Rats were placed in the recording room $30 \mathrm{~min}$ before testing. Each of the rats was placed in the center zone of an $\mathrm{OF}$ at the beginning and allowed it to explore the maze for $5 \mathrm{~min}$. The following behavioral elements were quantified: frequency of the central zone (CZ) entered and total movement in the recording cage. Anxiety-like behavior in the OF test is triggered by the agoraphobia (while rats were forced into the arena which is very large relative to the rats' nature). The low number of crossing the CZ demonstrates animal's anxiety (Prut and Belzung, 2003).

\section{Histological Identification}

Each animal was deeply anesthetized with sodium pentobarbital (60 mg/kg, i.p.). A transcardiac perfusion was performed with $0.9 \%$ saline followed by $3.5 \%$ paraformaldehyde in $0.1 \mathrm{M}$ phosphate buffer at $\mathrm{pH}$ 7.4. The brain was then removed and post-fixed in the same fixative with $20 \%$ sucrose overnight at $4^{\circ} \mathrm{C}$. Brain sections in the coronal plane were cut at $40 \mu \mathrm{m}$ thicknesses. The positions of electrode tips were plotted on sections of the rat atlas of Paxinos and Watson.

\section{Statistical Analysis}

The AD thresholds of the two group were assessed by the independent $t$ test. The correlation coefficient was used to evaluate the relationship between the $\mathrm{AD}$ threshold and the stimulated number attaining Racine's stage 5. Racine's score was assessed by 1-way repeated measures analysis of variance (ANOVA) on ranks. The AD durations and indexes of the SC test were evaluated by 2 -way repeated measures ANOVA with one factor repetition, if appropriate, followed by post hoc $t$ test with Bonferroni correction. Indexes of the EPM test, OF test, and FS test were assessed by the independent $t$ tests.

Another interesting information was how many animals were comorbid with severe anxio-depressive behaviors. The present study calculated the rat number of the kindle group that the behavioral indexes exceeded the mean $\pm 99 \%$ confidence interval of behavioral indexes of the control group. Data was expressed as the mean \pm standard error of the mean (SEM). A two-tailed significance level was set at $p<0.05$.

\section{RESULTS}

In the present study, 58 rats were used. Five rats did not complete the experiment due to de-attachment of dental cement from the head under amygdala kindling. ADs were unable to be elicited from three rats through amygdala kindling, which was due to electrode placement outside the basolateral nucleus of the amygdala. The histological data showed that electrode tips of 25 rats were located in the basolateral nuclei of the right amygdala (Figure 2). Finally, 50 rats (control $=25$, kindle $=25$ ) were used in the first experiment for further analysis. In the second experiment, 30 rats (control $=15$, kindle $=15$ ) were used to explore anxious state in terms of the OF test.

\section{Kindling}

The $\mathrm{AD}$ thresholds of the kindle and control groups were $153.0 \pm 27.3$ and $153.0 \pm 26.3 \mu \mathrm{A}$, respectively. AD thresholds of the two groups had no significant difference. The AD thresholds were in the range of 100-200 $\mu \mathrm{A}$, which was lower or equal to the current intensity of the rapid amygdala kindling.

Figure 3 shows the $\mathrm{AD}$ of the 40th stimulation in a rat. An extremely dynamic change in pattern and amplitude of waveforms was observed. Aberrant brain activity of $>400 \mu \mathrm{V}$ was observed, accompanied by a tonic-clonic convulsion 30-50 s after stimulation. Brain activity became a brief silence immediately after the AD then followed by postictal epileptiform discharge. The amygdala showed a strongly aberrant discharge compared to the cortex.

Throughout the rapid kindling process, rats progressively developed a tonic-clonic stage five convulsion as the stimulation number increased (Figure 4). All rats in the kindle group showed Racine's stage 5 (tonic-clonic convulsion) after 40 right-side amygdala stimulations. The Racine's score significantly increased as the stimulation number increased $\left(\chi^{2}=903, p<0.001\right)$. In addition, the $\mathrm{AD}$ duration became significantly longer as 

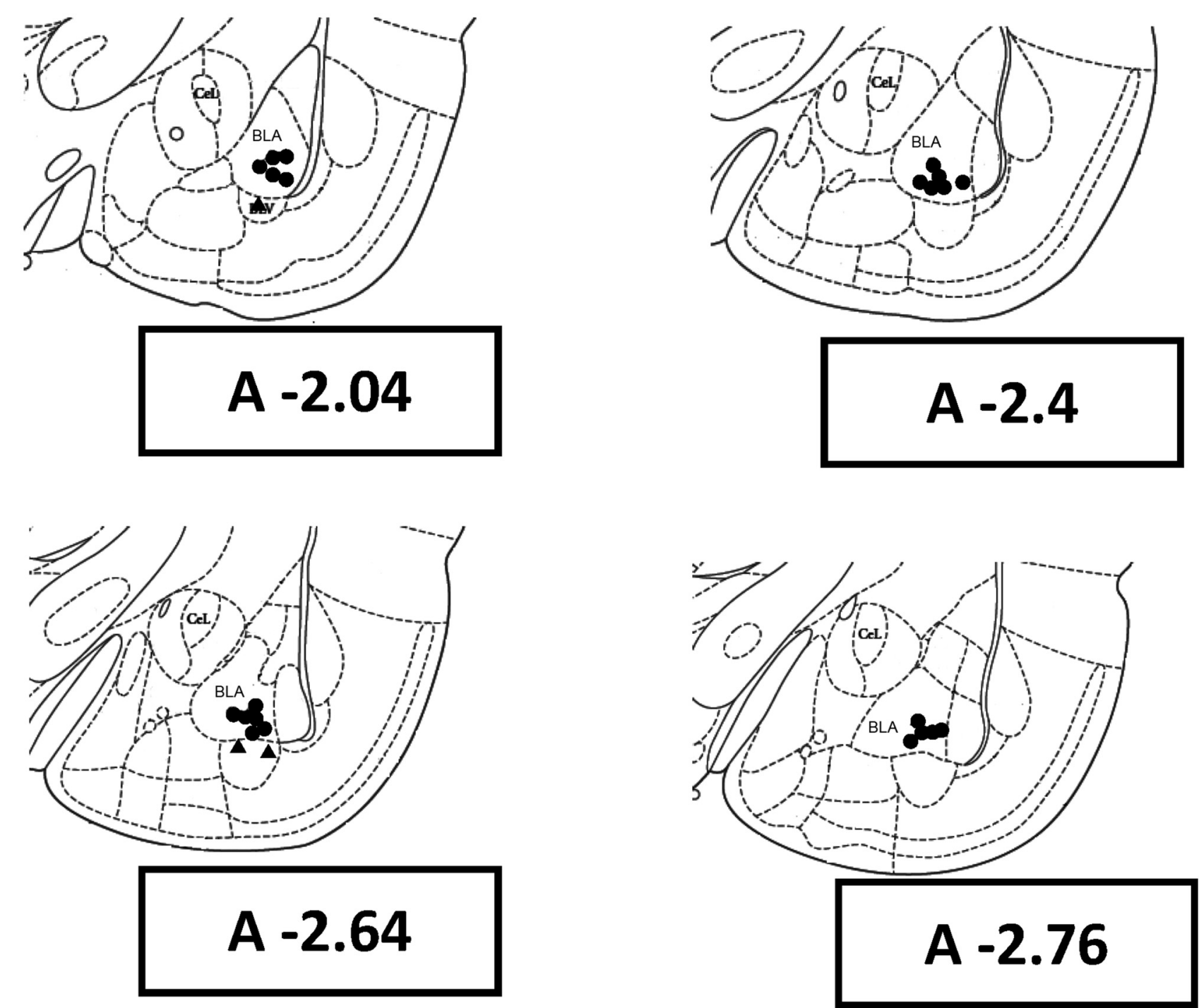

FIGURE 2 | Rat brain coronal sections with reference to the bregma show histological reconstruction of the stimulation sites in kindle animals. BLA, basolateral amygdaloid nucleus, anterior; CeL, centrolateral nucleus.

the amygdala kindling number increased $\left(F_{(39,936)}=334.7\right.$, $p<0.001)$.

Intuitively, rats with a low $\mathrm{AD}$ threshold might receive over-stimulation for a constant 200- $\mu$ A kindling, which might result in attaining Racine's stage 5 quickly. There was however no significant correlation between the $\mathrm{AD}$ threshold and the stimulating number attaining Racine's stage $5(r=-0.24$, $p=0.27)$.

\section{Sucrose Consumption Test}

Fluid intake during the SC test progressively increased as time passed in the two groups (Figure 5). Obviously, the kindle group had a significantly lower sucrose intake than that of the control group. Sucrose intake had significant differences in the factors of time $\left(F_{(4,249)}=228.0, p<0.001\right)$ and treatment
$\left(F_{(1,249)}=6.173, p=0.017\right)$. There was no difference in SC between the two groups on the first day, but the kindle group had a significantly lower sucrose intake than the control group for the following 4 days (Figure 5A). More than $40 \%$ of the kindle group showed a remarkable lower sucrose intake than the mean $-99 \%$ confidence interval of the control group in the last 4 days of the SC test (Table 1). The sucrose intake normalized by body weight showed significant differences in the factors of time $\left(F_{(4,249)}=199.2, p<0.001\right)$ and treatment $\left(F_{(1,249)}=4.885, p=0.032\right)$. The kindle group had a lower sucrose intake than the control group. In particular, there was no difference in normalized SC on the first day, but the kindle group showed significantly lower normalized sucrose intake than the control group at the $2 \mathrm{nd}$, 4 th, and 5 th days (Figure 5B). 


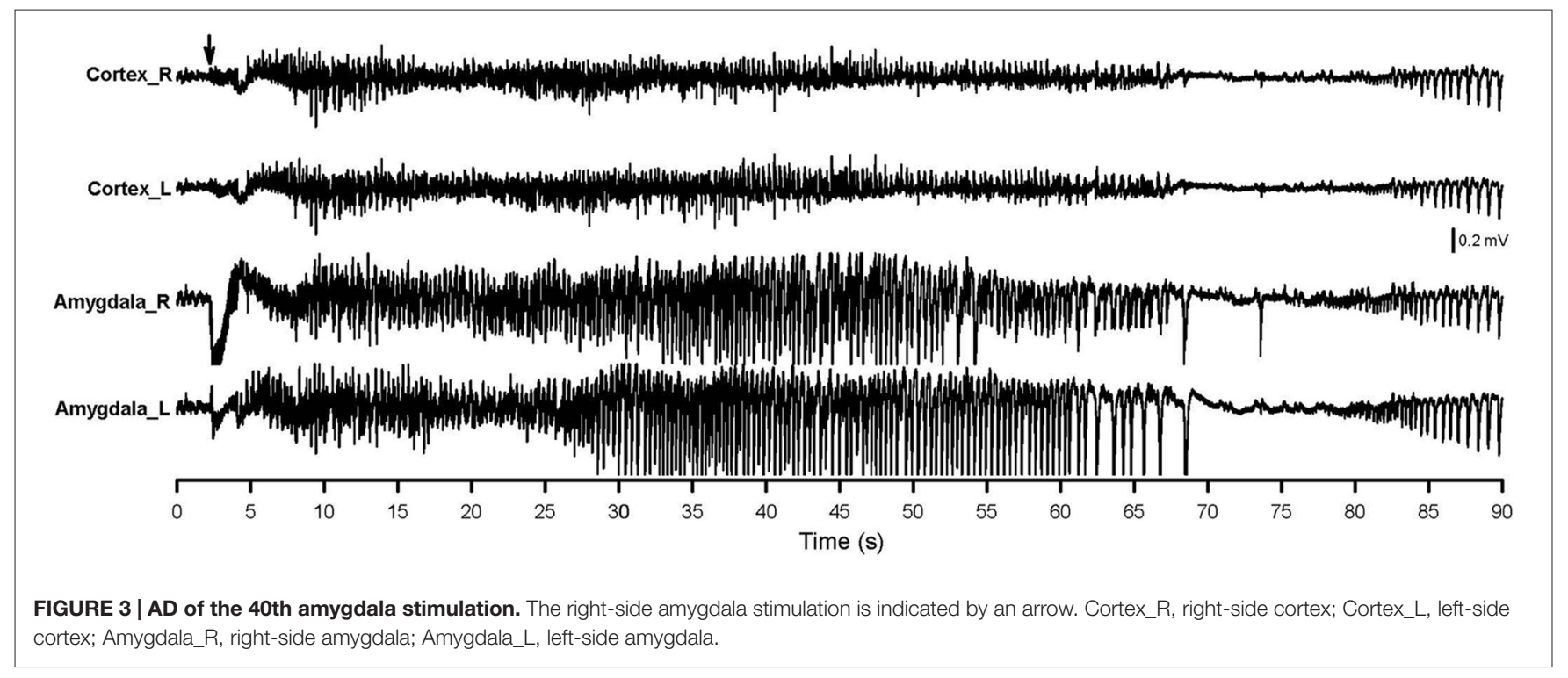

We also analyzed the approaching number during the SC test. The approaching number showed a significant difference in the factor of time $\left(F_{(4,249)}=167.4, p<0.001\right)$. The treatment factor almost attained a significant level $\left(F_{(1,249)}=3.835\right.$, $p=0.056)$. The kindle group had a remarkable lower frequency of approaching the sucrose bottle compared to the control group. In addition, the sucrose intake per approach made a significant difference in the factor of time $\left(F_{(4,249)}=59.291, p<0.001\right)$. The treatment factor didn't attain a significant level $\left(F_{(1,249)}=3.167\right.$, $p=0.081)$. The two groups had no difference in the SC per approach.

\section{Forced Swim Test}

Figure 6 shows indexes during the FS test. The kindle group showed a significantly longer immobility behavior compared to that of the control group $(t=5.334, p<0.001)$. Twenty rats $(80 \%)$ of the kindle group had longer immobility compared to the mean $+99 \%$ confidence interval of the control group (Table 1). The kindle group had a significantly shorter duration of active swimming than the control group $(t=5.72, p<0.001)$. The duration of the swimming was not different between the two groups $(t=0.703, p=0.486)$.

\section{Elevated Plus Maze Test}

Figure 7 shows the entry ratio of OAs/CAs and duration of staying OAs of an EPM. The kindle group had significantly shorter duration of staying in OAs than the control group $(t=2.068, p=0.044)$. The entry ratio into OAs and CAs between the two groups was significantly different $(t=2.062, p=0.044)$. More than $40 \%$ of the kindle group had lower value than the mean $-99 \%$ confidence interval of the control group (Table 1). Total movement in the EPM was not different between the two groups (control, $19.8 \pm 6.0 \mathrm{~m}$; kindle, $19.5 \pm 5.3 \mathrm{~m} ; t=0.137$, $p=0.89$ ).

\section{Open Field Test}

Figure 8 shows the frequency crossing the $\mathrm{CZ}$ of an $\mathrm{OF}$ cage. The kindle group had a significantly lower frequency crossing the CZ compared with the control group $(t=2.58$, $p=0.015)$. Forty percent of the kindle group fell out the range of the mean $-99 \%$ confidence interval of the control group (Table 1). Total movement in the OF apparatus showed no difference between the two groups (control, $29.5 \pm 2.4 \mathrm{~m}$; kindle, $29.1 \pm 2.1 \mathrm{~m} ; t=0.137$, $p=0.89)$.

\section{DISCUSSION}

The rapid amygdala kindling successully elicited ADs and afterwards caused a tonic-clonic motor seizure. The kindle group showed anxiety-like behavior in terms of low frequency entering open area of the EPM test and low frequency crossing $\mathrm{CZ}$ of the $\mathrm{OF}$ test. Moreover, the kindle group displayed low sucrose intake (anhedonia) in the SC test and long immobility (despair) in the FS test, which are indexes of depressive behaviors. Our results indicate that the rapid kindling of rightside amygdala aggravates anxiety- and depression-like behaviors. The rapid amygdala kindling model has a face validity as TLE patients.

Rats after the rapid amygdala kindling had the propensity to develop anxiety- and depression-like behaviors in terms of four behavioral tests. A considerable portion of patients with epilepsy experience cormobid anxiety (25-52\%) or depression (16-50\%) (Piazzini et al., 2001; Kanner, 2007; Desai et al., 2010; de Oliveira et al., 2010). In the current study, anxiety-like behavior occurred in $40-48 \%$ of the kindle group. Andedonia behavior showed in $40-48 \%$ of the kindle group, and despairlike behavior existed in $80 \%$ of the kindle group. There are similar prevalences to psychiatric comorbidity in patients with epilepsy and TLE-like model with rapid amygdala kindling. During the measurement period in the EPM or the OF, the 


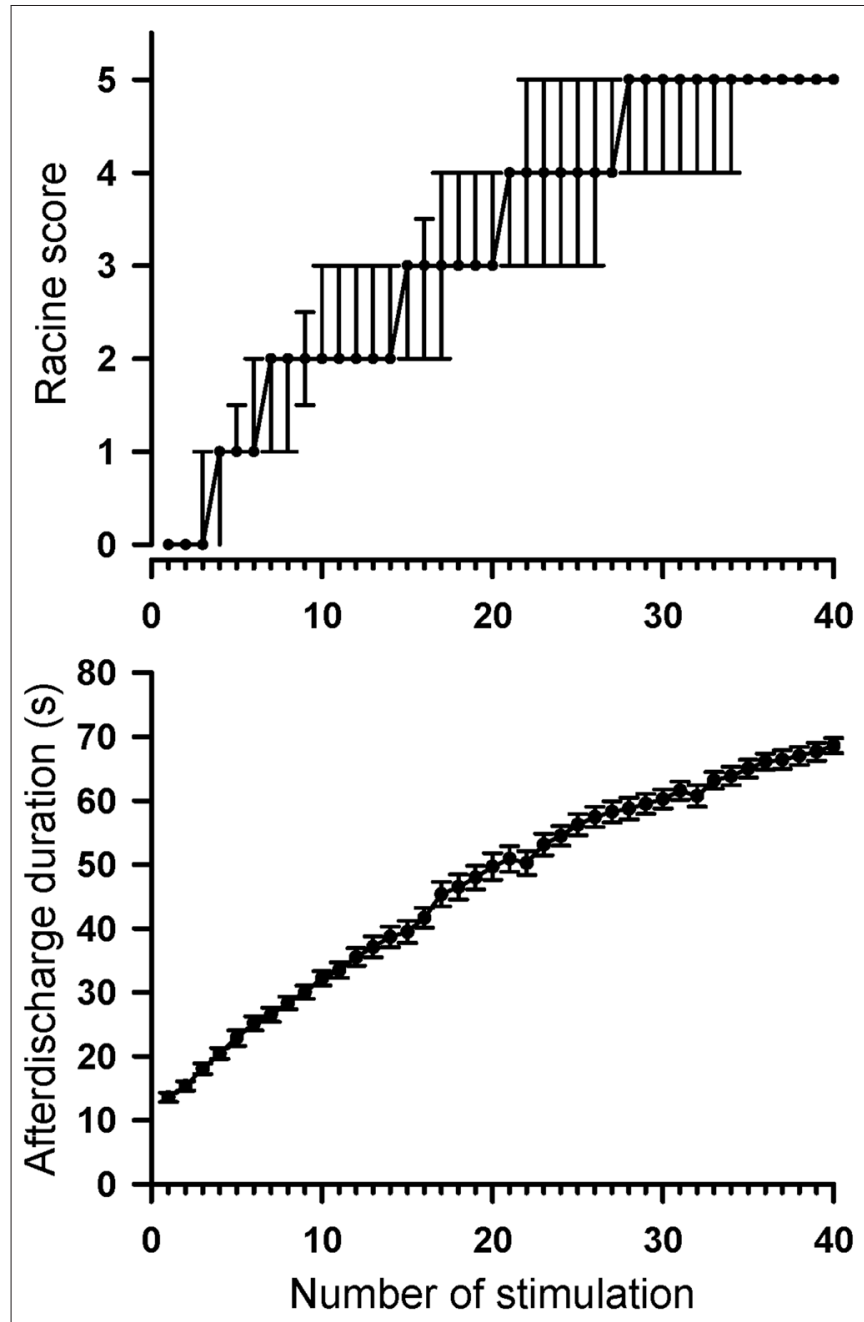

FIGURE 4 | Progression of Racine's seizure score and AD duration throughout $\mathbf{4 0}$ amygdala stimulations in the control and kindle groups. The data are presented with the median $\pm 25-75$ percentile on Racine's score and the Mean \pm SEM on AD duration.

total movement was not different in the two groups. Moreover, there was no change of normal swimming in the FS test, and exploring behavior (in terms of approach frequency) had no motor impairment in the SC test. These results suggest that the anxiety- and depression-like behaviors are related to the amygdala kindling.

Rapid amygdala kindling has the advantage that Racine's stage 5 can occur within the first few days because up to 24 stimulations can be given each day (Lothman et al., 1985; Lothman and Williamson, 1994). Increased number of kindling stimulations were accompanied by long $\mathrm{AD}$ duration of both the amygdala and frontal cortex, which indicates a neural plasticity taking place in the amygdala and associated network, such as fiber sprouting (Ebert and Löscher, 1995; Smith et al., 2005). The phenomenon of increased fiber sprouting (Kandratavicius et al., 2012) or abnormal functional connectivity in the amygdalofrontal network (Doucet et al., 2013) is also observed in TLE patients. The rapid kindling process has been used to assess anticonvulsants or alternative therapeutics (De Smedt et al., 2007; Shahpari et al., 2012; Shojaei et al., 2014). The present study showed psychiatric comorbidity in rats with the rapid amygdala kindling. The data provides additional validation of the rapid amygdala kindling as a TLE-like model.

In a previous study (Jones et al., 2009), early maternal separation of rats at birth accelerated the kindling process. The rapid amygdala kindling resulted in an anxiolytic effect as evidenced by an increase in time spent in the OAs of the EPM compared with the sham group. In contrast, the present study showed $\mathrm{AD}$ with severe motor seizure and anxiety-like behavior after the rapid amygdala kindling of normal rats with no stress of early separation. Stress of early isolation or maternal separation often causes abnormal development of the limbic region (Kumar et al., 2011). The irregular limbic modulation may alter the level of psychomotor behavior. It may be a reason to explain the different effects of the rapid kindling on rats.

The present study used a re-test stimulation $60 \mathrm{~min}$ before the EPM test or the OF test to ascertain seizure severity in the kindle group exclusively. Animals with a Racine's stage 5 motor seizure in response to the re-test stimulation were further analyzed. Because movement in either the EPM test or the OF test had no significant difference between the two groups, kindled animals thus have a low interest to enter OAs in the EPM test or to cross the $\mathrm{CZ}$ in the $\mathrm{OF}$ test. Before the anxiety behavioral tests, the re-test stimulation perhaps elicits a stressful event which may result in worsening the anxiety-like behavior. A further study needs to be done to determine the contribution of the re-test stimulation on anxiety-like behavior.

In our previous study, spike patterns of epileptiform discharges with regard to the severity of motor seizures have a similar development between conventional and rapid amygdala kindling (Wang et al., 2016). The development of $\mathrm{AD}$ and motor seizure stage seems to be similar in conventional kindling (Adamec and Morgan, 1994; Helfer et al., 1996) and rapid kindling (Jones et al., 2009) of the amygdala. An anxiogenic response is reported in the conventional amygdala kindling model (Adamec and Morgan, 1994; Helfer et al., 1996; Hannesson et al., 2008) or the pilocarpine- and kainic acid-induced TLE models (Inostroza et al., 2012). The effect of conventional amgdala kindling on anxiety-like behavior has been demonstrated to be related to stimulation side/site, gender, etc., (Adamec and Morgan, 1994; Kalynchuk, 2000; Wintink et al., 2003; Adamec et al., 2005). Furthermore, increases in emotionality or defensiveness are also accompanied by amygdala kindlings (Helfer et al., 1996). The reduction of exploring OAs of the EPM test in the amygdala kindling group is reversed by a benzodiazepam anxiolytic (Helfer et al., 1996). The amygdala is known as a fear and emotional circuit (Goddard, 1983). Accordingly, extensive stimulation of the amygdala causes dysregulations of the emotional network in convulsive animals. 


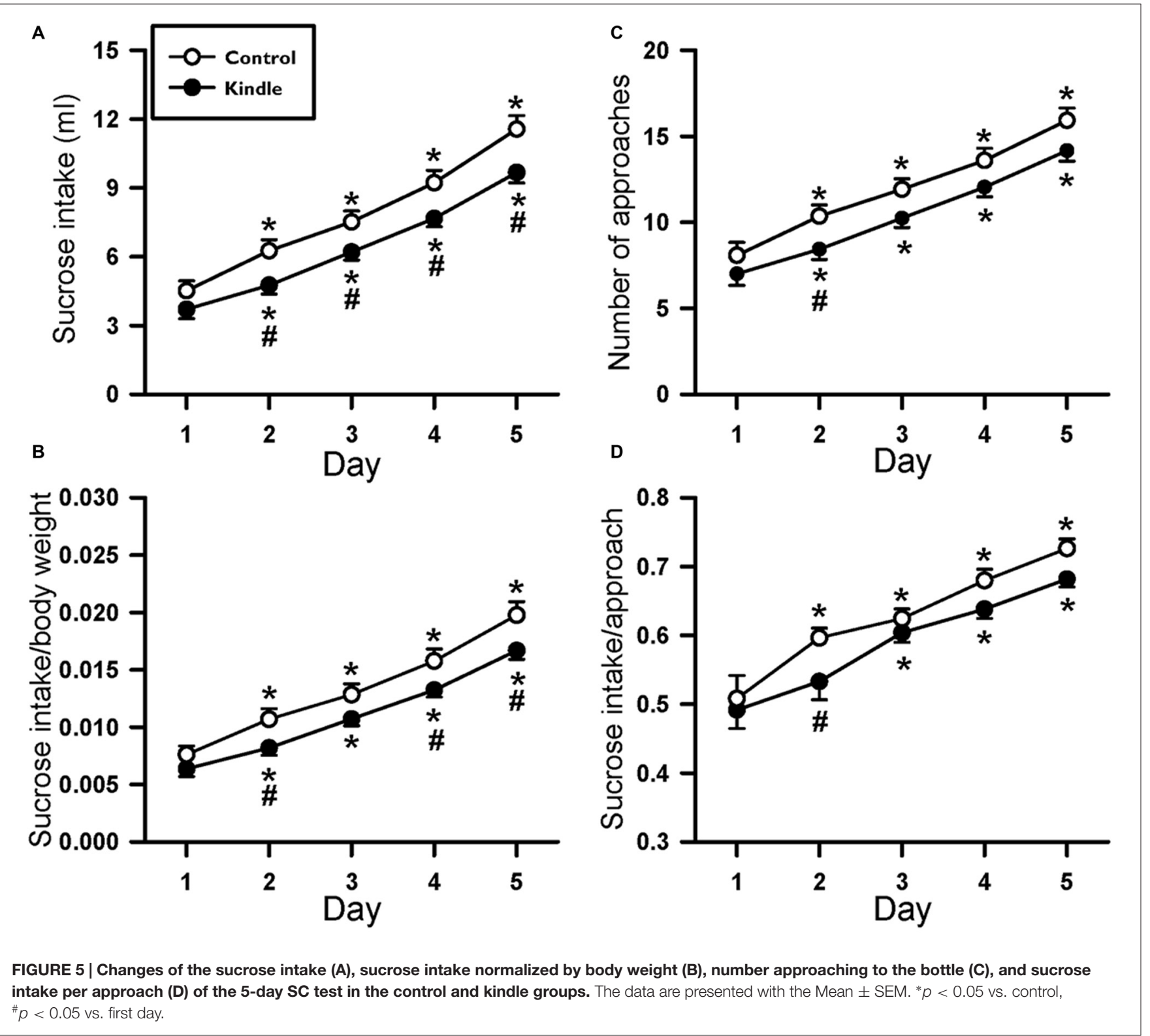

Patients with TLE are often comorbid with major depression (Kanner, 2007; Kandratavicius et al., 2012). The intake of sucrose fluid within $15 \mathrm{~min}$ in the 5-day SC test, which is validated as a hedonic measure (Jones et al., 2008; Huang et al., 2012), was significantly lower in the kindle group compared to the control group. Loss of taste preference and low consumption of saccharin solution is reported in immature rats under rapid amygdala kindling (Mazarati et al., 2007). The results support that the rapid kindling model should be associated with TLE patients' behaviors. However, an anhedonic phenomenon is debated in the pilocarpine- and kainic acid-induced TLE models (Mazarati et al., 2008; Inostroza et al., 2012; Tchekalarova et al., 2013; Klein et al., 2015). In contrast, conventional amygdala kindling had no significant difference in sucrose intake during the sucrose preference test (Helfer et al., 1996; Wintink et al., 2003; Adamec et al., 2004). Studies with insignificant findings only performed a 1-day measure of the sucrose intake. In this study, there was no significant difference at the first day in all indexes but revealed significance in the following days in the SC test (Figure 5). The pattern of changes of significance throughout the 5-day measures was also observed in the lamortrigine experiment (Huang et al., 2012). These results perhaps explain the controversial findings in the 1-day sucrose measure during previous studies.

The kindle group showed significantly longer immobility of the FS test compared to the control group. Immobility of the FS test is also related to seizure severity under a rapid kindling process (Mazarati et al., 2007). In a previous study (Helfer et al., 1996), immobility duration of the FS 
TABLE 1 | Rat number in the kindle group beyond the range (Mean $\pm 99 \%$ confidence interval) of the four behavioral tests of the control group.

\begin{tabular}{lc} 
Behavior & Number (ratio) \\
\hline Elevated plus maze test $(n=25)$ & \\
OA-duration & $10(40 \%)$ \\
OA/CA entry ratio & $12(48 \%)$ \\
Open field test $(n=15)$ & \\
$\quad$ Number crossing CZ & $6(40 \%)$ \\
Forced swim test $(n=25)$ & \\
$\quad$ Immobility & $20(80 \%)$ \\
Sucrose consumption test $(n=25)$ & \\
$\quad$ Sucrose intake (day1) & $10(40 \%)$ \\
Sucrose intake (day2) & $11(44 \%)$ \\
Sucrose intake (day3) & $12(48 \%)$ \\
Sucrose intake (day4) & $11(44 \%)$ \\
Sucrose intake (day5) & $12(48 \%)$
\end{tabular}

CA, closed arm; CZ, central zone; OA, open arm.

test in 10 right-amygdala kindled rats is higher but not significant than those of 10 non-kindled rats. A small sample size may cause low statistical power. No significant difference was reported in the immobility of the 10-min FS test whereas rats received kindling stimulations of the left amygdala (Wintink et al., 2003). The immobility of a 5-min FS test has been validated as an index of the despaired mood (Cryan et al., 2005; Huang et al., 2012). Kindled stimulations of the left-side and right-side amygdala produce different effects on anxiety-like behaviors (Adamec and Morgan, 1994; Adamec et al., 2005). Therefore, different measure duration of the FS test and stimulation site may account for the discrepancy between the present and previous studies. Normal swimming and active swimming in the FS test are increased by serotonergic and noradrenergic antidepressants, respectively (Cryan et al., 2005). The kindle group had an exclusive change in active swimming of the FS test, not in normal swimming. Our data may indicate that dysfunctions of noradrenergic neurons occur under rapid amygdala kindling.

A great portion (80\%) of the kindle group had longer immobility than the control group. The sensitivity of the FS test seemed to be 2-fold higher than the anhedonic portion ( $\sim 40 \%$ ) using the SC test. The distribution of the portion with kindling-induced depression-like behaviors in the two behavioral tests remarkably differs from the approximate portion of the same two behavioral tests in an acid-induced muscle pain model (Liu et al., 2014). The immobility in the FS test can reflect an actively coping strategy to an inescapable situation (Cryan et al., 2005). The immobility during the FS test is related to not only "behavioral despair" but also "learned helplessness". The "learned helplessness" usually leads to a loss of interest in work, life, etc. Afterward, individuals may evolve a feeling of hopelessness and reveal as immobility with sinking into water in the FS test with shorter duration of active swimmming in the kindle group (Figure 6). The results may partially support a several fold higher risk of suicide in patients with epilepsy (Kanner, 2007).

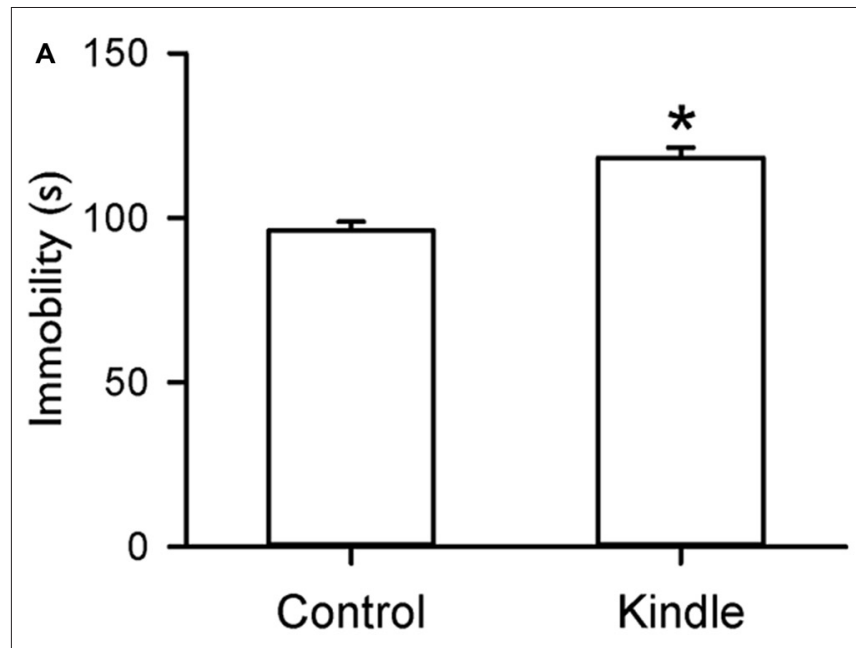

B

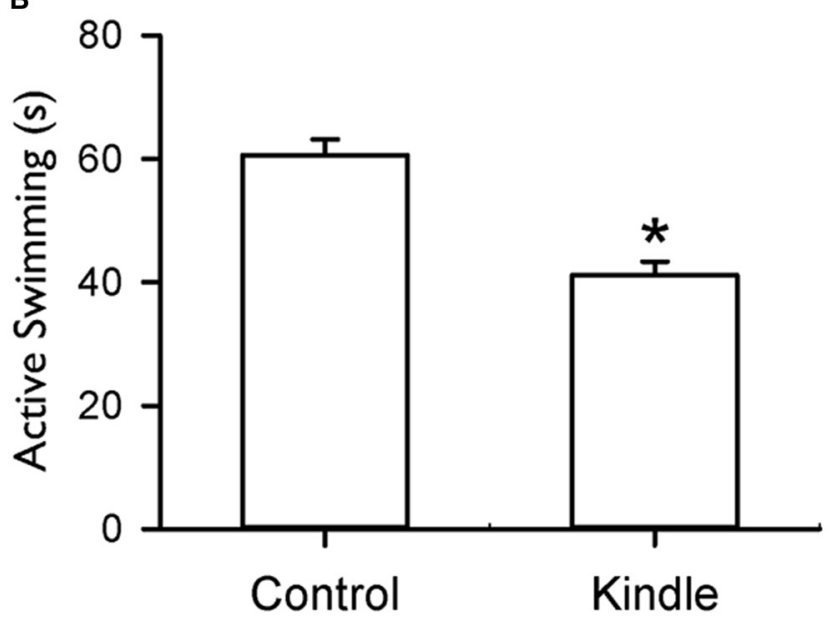

C

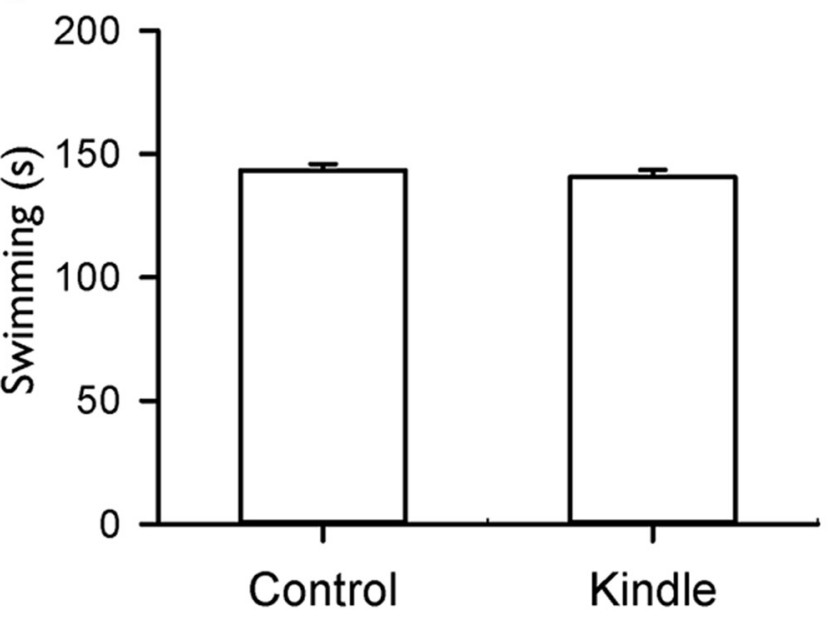

FIGURE 6 | Durations of immobility (A), active swimming (B) and swimming $(\mathrm{C})$ of the FS test in the control and kindle groups. The data are presented with the Mean \pm SEM. ${ }^{*} p<0.05$ vs. control.

The present study used adult rats of 8-15 weeks old. The functional brain changes or neurodegenerative progression is related with age. The amygdala kindling threshold of 

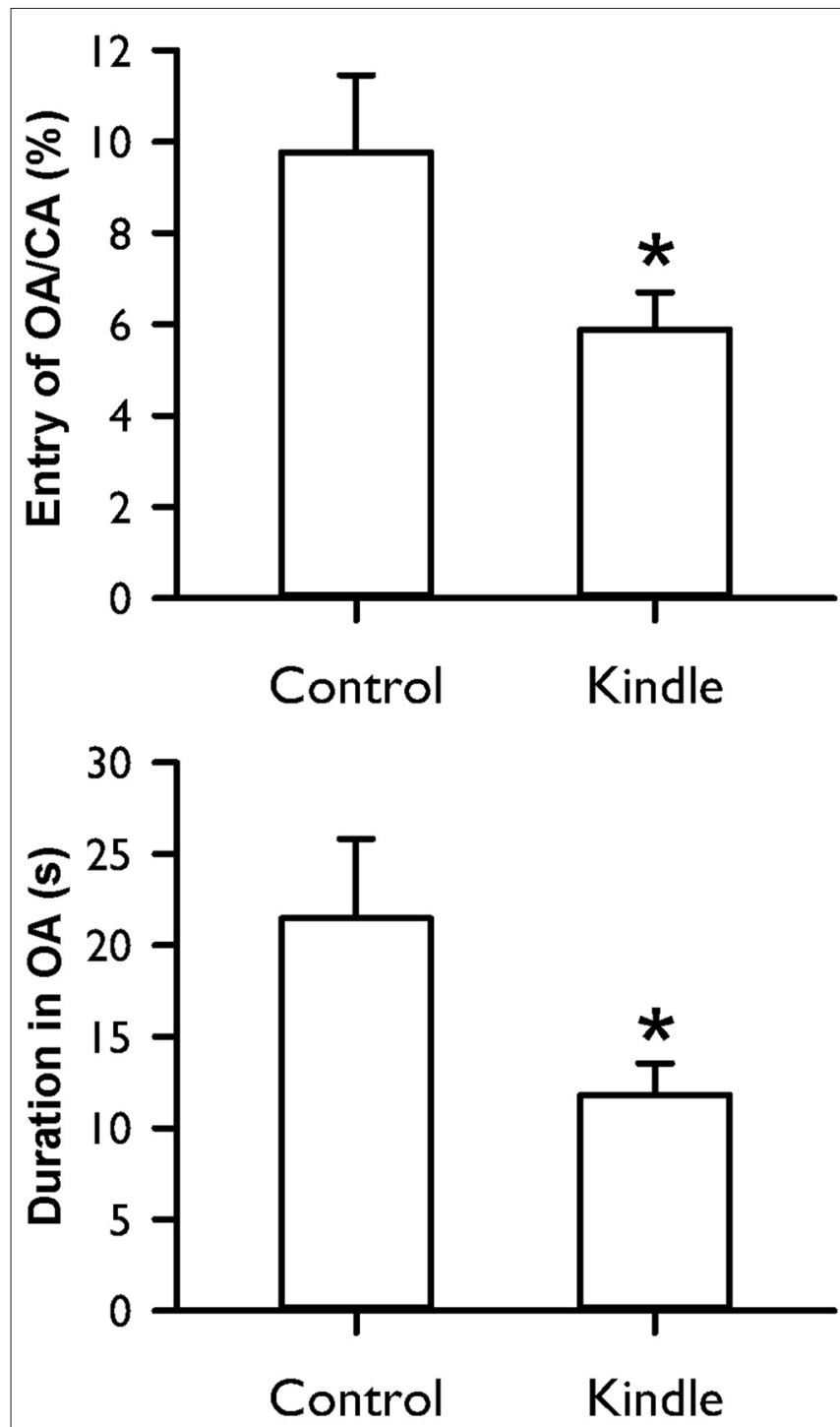

FIGURE 7 | Entry ratio of open arms (OAs)/closed arms (CAs) and duration of staying in OAs of the EPM test in the control and kindle groups. The data are presented with the Mean \pm SEM. ${ }^{*} p<0.05$ vs. control.

adult rats is higher than that of immature animals (Moshe et al., 1981). The kindling-induced motor behaviors in adult rats are more reliable than those of neonate or infant rats (Gilbert and Cain, 1981; Yoshioka et al., 2000). Although the present study used animals with a large variance in age, we showed consistent kindling phenomenon and also observed severe anxiety-like or depressive-like comorbidity in a considerable portion of rats. So as to strengthen the effect of kindling on psychiatric comorbidity further, animals with a small age range may be helpful to elevate statistical power.

Several aspects of consistencies are found between the rapid kindling and conventional kindling, including $\mathrm{AD}$ progression (Wang et al., 2016) and anxiogenic effect (Adamec and Morgan, 1994; Helfer et al., 1996). However, there were subtle differences,

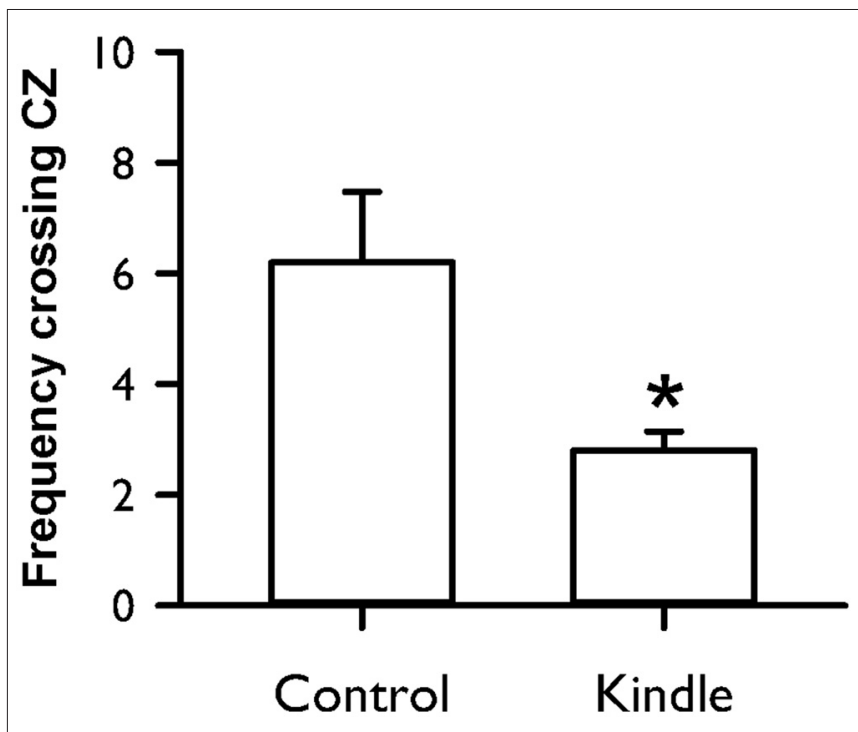

FIGURE 8 | Frequency crossing the central zone (CZ) of an OF in the control and kindle groups. The data are presented with the Mean $\pm \mathrm{SEM}$. ${ }^{*} p<0.05$ vs. control.

for example, fiber sprouting (Ebert and Löscher, 1995). Different observations were also found between the rapid kindling and conventional kindling in the measure of depression-like behavior, such as sucrose intake (Helfer et al., 1996; Wintink et al., 2003; Adamec et al., 2004) and immobility during the FS test (Helfer et al., 1996). As we mentioned above, those studies have different measure parameters or small sample numbers, which may cause varied results. To further clarify the effect of amygdala kindling on depressive comorbidity, it will be important to assess same depresion-like behavior tests in animals with rapid kindling or conventional kindling in the future.

In summary, rapid amygdala kindling elicited a tonic-clonic stage 5 convulsive seizure. The current study provides further evidence on the propensity of anxiety- and depression-like behaviors in a considerable portion of the TLE-like model with rapid amygdala kindling.

\section{AUTHOR CONTRIBUTIONS}

S-DC: experimental design and writing of the article. Y-LW and S-FL: data analysis. F-ZS: experimental design, data analysis, and article draft.

\section{ACKNOWLEDGMENTS}

The authors thank Ms. Yu-Hsing Huang on technical support and animal preparation. This work was supported by the Ministry of Science and Technology (NSC102-2220-E-006-002 and MOST103-2314-B-006-021-MY2). This study was partially supported by the "Aim for the Top University Plan" of the National Chiao Tung University and Ministry of Education, Taiwan. 


\section{REFERENCES}

Adamec, R., Blundell, J., and Burton, P. (2004). Anxiolytic effects of kindling role of anatomical location of the kindling electrode in response to kindling of the right basolateral amygdala. Brain Res. 1024, 44-58. doi: 10.1016/j.brainres. 2004.06.074

Adamec, R. E., and Morgan, H. D. (1994). The effect of kindling of different nuclei in the left and right amygdala on anxiety in the rat. Physiol. Behav. 55, 1-12. doi: 10.1016/0031-9384(94)90002-7

Adamec, R., Shallow, T., and Burton, P. (2005). Anxiolytic and anxiogenic effects of kindling-role of baseline anxiety and anatomical location of the kindling electrode in response to kindling of the right and left basolateral amygdala. Behav. Brain Res. 159, 73-88. doi: 10.1016/j.bbr.2004.10.004

Cryan, J. F., Valentino, R. J., and Lucki, I. (2005). Assessing substrates underlying the behavioral effects of antidepressants using the modified rat forced swimming test. Neurosci. Biobehav. Rev. 29, 547-569. doi: 10.1016/j.neubiorev. 2005.03.008

de Oliveira, G. N., Kummer, A., Salgado, J. V., Portela, E. J., Sousa-Pereira, S. R., David, A. S., et al. (2010). Psychiatric disorders in temporal lobe epilepsy: an overview from a tertiary service in Brazil. Seizure 19, 479-484. doi: 10.1016/j. seizure.2010.07.004

De Smedt, T., De Rouck, S., Raedt, R., Wyckhuys, T., Waterschoot, L., De Herdt, V., et al. (2007). Serial day rapid kindling is an effective tool in screening the anti-epileptic properties of topiramate. Seizure 16, 620-626. doi: 10.1016/j. seizure.2007.04.013

Desai, S. D., Shukla, G., Goyal, V., Singh, S., Padma, M. V., Tripathi, M., et al. (2010). Study of DSM-IV Axis I psychiatric disorders in patients with refractory complex partial seizures using a short structured clinical interview. Epilepsy Behav. 19, 301-305. doi: 10.1016/j.yebeh.2010.07.005

Doucet, G. E., Skidmore, C., Sharan, A. D., Sperling, M. R., and Tracy, J. I. (2013). Functional connectivity abnormalities vary by amygdala subdivision and are associated with psychiatric symptoms in unilateral temporal epilepsy. Brain Cogn. 83, 171-182. doi: 10.1016/j.bandc.2013.08.001

Ebert, U., and Löscher, W. (1995). Differences in mossy fibre sprouting during conventional and rapid amygdala kindling of the rat. Neurosci. Lett. 190, 199-202. doi: 10.1016/0304-3940(95)11540-d

Ehlers, C. L., and Koob, G. F. (1985). Locomotor behavior following kindling in three different brain sites. Brain Res. 326, 71-79. doi: 10.1016/00068993(85)91385-x

Gilbert, M. E., and Cain, D. P. (1981). A developmental study of kindling in the rat. Brain Res. 254, 321-328. doi: 10.1016/0165-3806(81)90041-9

Goddard, G. V. (1983). The kindling model of epilepsy. Trend Neurosci. 6, 275-279. doi: 10.1016/0166-2236(83)90118-2

Hannesson, D. K., Pollock, M. S., Howland, J. G., Mohapel, P., Wallace, A. E., and Corcoran, M. E. (2008). Amygdaloid kindling is anxiogenic but fails to alter object recognition or spatial working memory in rats. Epilepsy Behav. 13, 52-61. doi: 10.1016/j.yebeh.2008.02.007

Helfer, V., Deransart, C., Marescaux, C., and Depaulis, A. (1996). Amygdala kindling in the rat: anxiogenic-like consequences. Neuroscience 73, 971-978. doi: 10.1016/0306-4522(96)00081-4

Huang, H. Y., Lee, H. W., Chen, S. D., and Shaw, F. Z. (2012). Lamotrigine ameliorates seizures and psychiatric comorbidity in a rat model of spontaneous absence epilepsy. Epilepsia 53, 2005-2014. doi: 10.1111/j.1528-1167.2012. 03664.x

Inostroza, M., Cid, E., Menendez de la Prida, L., and Sandi, C. (2012). Different emotional disturbances in two experimental models of temporal lobe epilepsy in rats. PLoS One 7:e38959. doi: 10.1371/journal.pone.0038959

Jones, N. C., Kumar, G., O’Brien, T. J., Morris, M. J., Rees, S. M., and Salzberg, M. R. (2009). Anxiolytic effects of rapid amygdala kindling and the influence of early life experience in rats. Behav. Brain Res. 203, 81-87. doi: 10. 1016/j.bbr.2009.04.023

Jones, N. C., Salzberg, M. R., Kumar, G., Couper, A., Morris, M. J., and O'Brien, T. J. (2008). Elevated anxiety and depressive-like behavior in a rat model of genetic generalized epilepsy suggesting common causation. Exp. Neurol. 209, 254-260. doi: 10.1016/j.expneurol.2007.09.026

Kalynchuk, L. E. (2000). Long-term amygdala kindling in rats as a model for the study of interictal emotionality in temporal lobe epilepsy. Neurosci. Biobehav. Rev. 24, 691-704. doi: 10.1016/s0149-7634(00)00031-2
Kandratavicius, L., Hallak, J. E., Young, L. T., Assirati, J. A., Carlotti, C. G. Jr., Leite, J. P., et al. (2012). Differential aberrant sprouting in temporal lobe epilepsy with psychiatric co-morbidities. Psychiatry Res. 195, 144-150. doi: 10. 1016/j.psychres.2011.06.005

Kanner, A. M. (2007). Epilepsy and mood disorders. Epilepsia 48, 20-22. doi: 10. 1111/j.1528-1167.2007.01395.x

Klein, S., Bankstahl, J. P., Loscher, W., and Bankstahl, M. (2015). Sucrose consumption test reveals pharmacoresistant depression-associated behavior in two mouse models of temporal lobe epilepsy. Exp. Neurol. 263, 263-271. doi: 10.1016/j.expneurol.2014.09.004

Kumar, G., Jones, N. C., Morris, M. J., Rees, S., O’Brien, T. J., and Salzberg, M. R. (2011). Early life stress enhancement of limbic epileptogenesis in adult rats: mechanistic insights. PLoS One 6:e24033. doi: 10.1371/journal.pone. 0024033

Liu, Y. T., Shao, Y. W., Yen, C. T., and Shaw, F. Z. (2014). Acid-induced hyperalgesia and anxio-depressive comorbidity in rats. Physiol. Behav. 131, 105-110. doi: 10.1016/j.physbeh.2014.03.030

Lothman, E. W., Hatlelid, J. M., Zorumski, C. F., Conry, J. A., Moon, P. F., and Perlin, J. B. (1985). Kindling with rapidly recurring hippocampal seizures. Brain Res. 360, 83-91. doi: 10.1016/0006-8993(85)91223-5

Lothman, E. W., and Williamson, J. M. (1994). Closely spaced recurrent hippocampal seizures elicit two types of heightened epileptogenesis: a rapidly developing, transient kindling and a slowly developing, enduring kindling. Brain Res. 649, 71-84. doi: 10.1016/0006-8993(94)91050-2

Mazarati, A., Shin, D., Auvin, S., Caplan, R., and Sankar, R. (2007). Kindling epileptogenesis in immature rats leads to persistent depressive behavior. Epilepsy Behav. 10, 377-383. doi: 10.1016/j.yebeh.2007.02.001

Mazarati, A., Siddarth, P., Baldwin, R. A., Shin, D., Caplan, R., and Sankar, R. (2008). Depression after status epilepticus: behavioural and biochemical deficits and effects of fluoxetine. Brain 131, 2071-2083. doi: 10.1093/brain/ awn 117

Morimoto, K., Fahnestock, M., and Racine, R. J. (2004). Kindling and status epilepticus models of epilepsy: rewiring the brain. Prog. Neurobiol. 73, 1-60. doi: 10.1016/j.pneurobio.2004.03.009

Moshe, S. L., Sharpless, N. S., and Kaplan, J. (1981). Kindling in developing rats: variability of afterdischarge thresholds with age. Brain Res. 211, 190-195. doi: 10.1016/0006-8993(81)90082-2

Piazzini, A., Canevini, M. P., Maggiori, G., and Canger, R. (2001). Depression and anxiety in patients with epilepsy. Epilepsy Behav. 2, 481-489. doi: 10.1006/ebeh. 2001.0247

Pitkänen, A., Tuunanen, J., Kälviäinen, R., Partanen, K., and Salmenperä, T. (1998). Amygdala damage in experimental and human temporal lobe epilepsy. Epilepsy Res. 32, 233-253. doi: 10.1016/s0920-1211(98)00055-2

Prut, L., and Belzung, C. (2003). The open field as a paradigm to measure the effects of drugs on anxiety-like behaviors: a review. Eur. J. Pharmacol. 463 3-33. doi: 10.1016/s0014-2999(03)01272-x

Racine, R. J. (1972). Modification of seizure activity by electrical stimulation. II. Motor seizure. Electroencephalogr. Clin. Neurophysiol. 32, 281-294. doi: 10. 1016/0013-4694(72)90177-0

Racine, R. J., Burnham, W. M., Gartner, J. G., and Levitan, D. (1973). Rates of motor seizure development in rats subjected to electrical brain stimulation: strain and inter-stimulation interval effects. Electroencephalogr. Clin. Neurophysiol. 35, 553-556. doi: 10.1016/0013-4694(73)90033-3

Rodgers, R. J., and Dalvi, A. (1997). Anxiety, defence and the elevated plus-maze. Neurosci. Biobehav. Rev. 21, 801-810. doi: 10.1016/s0149-7634(96) 00058-9

Shahpari, M., Mirnajafi-Zadeh, J., Firoozabadi, S. M., and Yadollahpour, A. (2012). Effect of low-frequency electrical stimulation parameters on its anticonvulsant action during rapid perforant path kindling in rat. Epilepsy Res. 99, 69-77. doi: $10.1016 /$ j.eplepsyres.2011.10.023

Shaw, F. Z., Lai, C. J., and Chiu, T. H. (2002). A low-noise flexible integrated system for recording and analysis of multiple electrical signals during sleep-wake states in rats. J. Neurosci. Methods 118, 77-87. doi: 10.1016/s0165-0270(02) 00146-2

Shaw, F. Z., Yen, C. T., and Chen, R. F. (2003). A simple and effective process for noise reduction of multichannel cortical field potential recordings in freely moving rats. J. Neurosci. Methods 124, 167-174. doi: 10.1016/s01650270(03)00005-0 
Shojaei, A., Semnanian, S., Janahmadi, M., Moradi-Chameh, H., Firoozabadi, S. M., and Mirnajafi-Zadeh, J. (2014). Repeated transcranial magnetic stimulation prevents kindling-induced changes in electrophysiological properties of rat hippocampal CA1 pyramidal neurons. Neuroscience 280, 181-192. doi: 10.1016/j.neuroscience.2014.09.022

Smith, P. D., McLean, K. J., Murphy, M. A., Turnley, A. M., and Cook, M. J. (2005). Seizures, not hippocampal neuronal death, provoke neurogenesis in a mouse rapid electrical amygdala kindling model of seizures. Neuroscience 136, 405-415. doi: 10.1016/j.neuroscience.2005.07.055

Tchekalarova, J., Petkova, Z., Pechlivanova, D., Moyanova, S., Kortenska, L., Mitreva, R., et al. (2013). Prophylactic treatment with melatonin after status epilepticus: effects on epileptogenesis, neuronal damage and behavioral changes in a kainate model of temporal lobe epilepsy. Epilepsy Behav. 27, 174-187. doi: 10.1016/j.yebeh.2013.01.009

Wang, Y. L., Chen, Y. L., Su, A. W., Shaw, F. Z., and Liang, S. F. (2016). Epileptic pattern recognition and discovery of the local field potential in amygdala kindling process. IEEE Trans. Neural Syst. Rehabil. Eng. 24, 374-385. doi: 10. 1109/TNSRE.2015.2512258
Wintink, A. J., Young, N. A., Davis, A. C., Gregus, A., and Kalynchuk, L. E. (2003). Kindling-induced emotional behavior in male and female rats. Behav. Neurosci. 117, 632-640. doi: 10.1037/0735-7044.117.3.632

Yoshioka, S., Mitani, H., Maeda, K., Takeo, S., Matsuda, K., Katayama, S., et al. (2000). Age-specific effects of noradrenergic alpha-2 agonist clonidine on the development of amygdaloid kindling in developing rats. Brain Res. Dev. Brain Res. 119, 283-288. doi: 10.1016/s0165-3806(99)00179-0

Conflict of Interest Statement: The authors declare that the research was conducted in the absence of any commercial or financial relationships that could be construed as a potential conflict of interest.

Copyright (C) 2016 Chen, Wang, Liang and Shaw. This is an open-access article distributed under the terms of the Creative Commons Attribution License (CC BY). The use, distribution and reproduction in other forums is permitted, provided the original author(s) or licensor are credited and that the original publication in this journal is cited, in accordance with accepted academic practice. No use, distribution or reproduction is permitted which does not comply with these terms. 